\title{
30 Jahre AfnP-Symposium in Fulda Wir sehen uns am 25. und 26. Oktober
}

Das AfnP-Symposium in Fulda steht vor der Tür und wir freuen uns auf viele spannende Vorträge, interessante Workshops und regen Austausch mit allen Berufsgruppen aus dem Arbeitsfeld der Nephrologie. Besucher können bis zu 15 ärztliche Registrierungspunkte bekommen. Mitglieder der AfnP erhalten mit dieser Ausgabe gleichzeitig die Symposiums-CD mit den Vorträgen oder Abstracts. Bereits zum 3. Mal wird dieses Jahr der Förderpreis Nephrologische Pflege vergeben.

Am Samstag, den 25. und Sonntag, den 26. Oktober 2008 findet im Stadtsaal Fulda (Orangerie) das 30. AfnP-Symposium statt. Seit einigen Jahren erhalten die Ärzte und Pflegekräfte für den Besuch der Veranstaltung Registrierungs-

\section{Mitgliederversammlung}

Die AfnP-Mitgliederversammlung findet am Samstag, den 25.10.08 ab 17.30 Uhr im Stadtsaal Fulda statt.

Bitte kommen sie recht zahlreich und weisen Sie sich mit Ihrer Mitgliedskarte und Ausweis an der Tür aus. punkte. Die dazugehörigen Unterschriftenlisten, Einzelbescheinigungen und weitere Informationen erhalten Sie am AfnP-Stand.

Es finden in 6 Räumen parallel Vorträge statt. Aus organisatorischen Gründen kommt es am Sonntag im Raum Berlin und im Raum Köln zu Zeitverschiebungen. Das aktuelle Programm finden Sie auf unserer Homepage. Da die Veranstaltung und auch das Parallelprogramm immer weiter wachsen, müssen wir in diesem Jahr das Foyer im Hotel Maritim vollständig mit Standflächen belegen. So sind alle Flächen im und um das Maritimhotel und den Stadtsaal Fulda fest in AfnP-Hand.
Für das 31. Symposium am 24. und 25.10.2009 haben bereits wieder fast alle Aussteller zugesagt und da wir keine weiteren freien Flächen haben, denken wir über ein zusätzliches Zelt oder eine Ausstellungsflächenbegrenzung nach. Dies werden wir beim diesjährigen Industriemeeting am 26.10.2008 mit allen Beteiligten besprechen. Den Flyer mit einem ersten Programmentwurf für 2009 erhalten Sie in Fulda oder im Internet unter www.afnp.de.

Förderpreis Nephrologische Pflege Am Sonntag, den 26.10.2008 um 09.00 Uhr überreichen wir wieder zusammen mit dem Georg Thieme Verlag den Förderpreis Nephrologische Pflege im Stadtsaal Fulda. Dieses Jahr wurden deutlich mehr Arbeiten eingereicht und auch die Qualität der Arbeiten ist noch einmal deutlich gestiegen. Dies hat die Bewertung nicht einfacher gemacht. Insgesamt wurden Arbeiten aus 5 nephrologischen Schulen und eine unabhängige Einzelarbeit eingereicht.

Gewonnen hat die Arbeit „Angehörigenintegration am Beispiel dialysepflichtiger Patienten einer ambulanten Einrichtung“. Diese Arbeit hat alle Jurymitglieder überzeugt. Den 2. Platz teilen sich 2 Arbeiten. Alles Weitere in einer der nächsten Ausgaben der Dialyse aktuell.

\section{Eintrittskarten bekommen Sie Freitag ab 17 Uhr}

Bitte denken Sie daran, sich rechzeitig Ihre Eintrittskarte und Workshopkarte zu besorgen. Die Kasse für Mitglieder und Nichtmitglieder befindet sich im Foyer des Hotel Maritim. Die Kasse ist am Freitag von 17.00-20.00 Uhr und Samstag ab 07.30 Uhr geöffnet. Nichtmitglieder zahlen für beide Tage 50 Euro. Es ist nur Barzahlung möglich. AfnP-Mitglieder erhalten eine personenbezogene freie Eintrittskarte. Bitte halten Sie ihre Mitgliedskarte und Ihren Ausweis bereit.

\section{Die CD mit den Vorträgen ist für} Mitglieder in diesem Heft

Alle Nichtmitglieder können die CD mit den Vorträgen des 30. Symposiums am AfnP-Stand oder an der Kasse während des Symposiums für 5 Euro erwerben oder bei der AfnP bestellen (info@afnp. de). Wir müssen dann 2 Euro zusätzlich für Porto und Versand erheben.

Bis bald in Fulda!

Ihre Marion Bundschu 